\title{
Freeze Dead Line Algorithm for Vertical Handoff in Heterogeneous Wireless Networks
}

\author{
Hyeon-Jin Jeong and Seung-Sik Choi \\ Computer Engineering Department, Incheon National University, (Songdo-dong) \\ 119 Academy-ro, Yeonsu-gu, Incheon, Korea \\ \{oasishjj, sschoi\}@incheon.ac.kr
}

\begin{abstract}
In this paper, we propose an algorithm to solve handover problems in heterogeneous wireless networks. Many previous studies concentrated on the lower level layer to solve handover problems. In this paper, we concentrate on high level handover problems such as network and transport layers. We solve handover problems of the transport layer in a heterogeneous wireless networks and propose a Freeze DeadLine (FDL) algorithm for better performance. The proposed method is analyzed from a theoretical perspective and is verified using the Network Simulator- 2.
\end{abstract}

Keywords: Vertical handoff, FDL, Heterogeneous Wireless Networks

\section{Introduction}

Long Term Evolution Advance (LTE-A) has recently been launched on a commercial scale in Korea. In theory, LTE-A has a rapid network transmission speed of about $150 \mathrm{Mbps}$. This wireless technique is a significant development over the previous $3 \mathrm{G}$ networks. However, wireless networks require more than just the best transmission speed, they must also interact with others networks in each environments. The latest mobile terminal therefore offers many networks and not just a single network, as shown Figure 1. This is termed Heterogeneous Wireless and is based on many kinds of wireless networks gathered in the same environment, where each network offers a service to each user specific to their purposes.

However, heterogeneous wireless networks have some problems, including the Hand-off problems. In a heterogeneous wireless environment, users continuously move and select networks for their particular purpose. Heterogeneous wireless networks also have each special attributes as well, so Hand-off problems have to be remedied. Many studies are now addressing physical and link layer problems in hand-off. One of many proposed solutions is the 802.21 MIH standard [1]. The MIH standard serves hand-off solutions for the bottom and upper layers, separately, but concentrates mainly on the bottom layer. Consequently, MIH does not solve transport layer problems such as TCP-Timeout or re-transmission problems [2].

Other studies have addressed hand-off problems that are not physical and involve the upper layer rather than the link layer [3-6]. One main study investigated Freeze-TCP [3], and solved many transport layer hand-off problems, at least in theory. Other problems arose, however, when Freeze-TCP was applied to wireless networks. One issue is that Freeze-TCP cannot recognize when networks are frozen. If Freeze-TCP freezes networks too soon, this creates waste of networks bandwidth. If Freeze-TCP freezes networks too late, freeze request packet is lost and this causes useless Time-out and packet re-transmission.

Another proposed solution is Cross-layer methods [4, 5], which deliver some information from the bottom layer to the upper layer. 
Hand-off [6] is the process of moving a node from one network to other networks in a cellular or wireless network. Two types of hand-off are available; horizontal and vertical hand off. Horizontal hand-off occurs when the nodes move from the current network to homogeneous networks, while vertical hand-off occurs when the nodes move to heterogeneous networks. Vertical hand-off can occur as downward or upward vertical handoff. In downward vertical hand-off, the nodes move from low throughput, wide range networks to high throughput, short range networks. The nodes undergoing downward vertical hand-off, need more time to use full bandwidth because the bandwidth is abruptly increased. Upward vertical hand-off occurs when the nodes move from high throughput, short range networks to low throughput, wide range networks. Many packet losses occur in upward handoff because the nodes cannot adapt to the changed network.

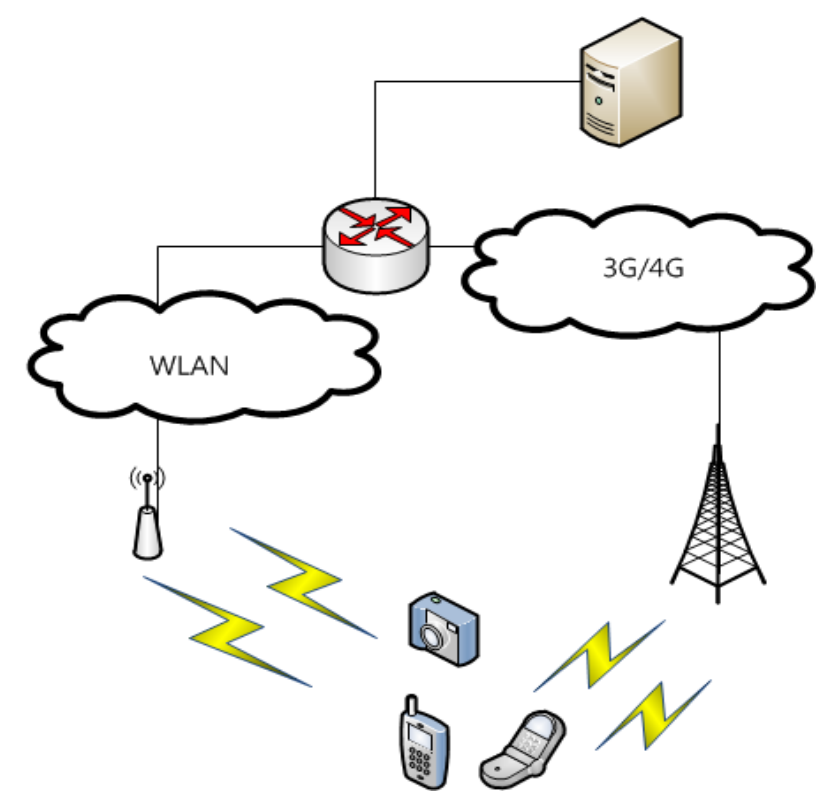

Figure 1. An Example of Heterogeneous Wireless Networks

In this paper, we propose an algorithm we call the Freeze DeadLine (FDL) method to solve hand-off problems in heterogeneous wireless networks when a freeze is initiated. FDL decreases the number of packet losses and provides better performance for exact freeze timing.

\section{Related Research}

Freeze - TCP [3] is a method for improving throughput in a hand-off situation. If a node is aware that hand-off has occurred, it sends a message to the server that sets the window size to be " 0 ". When the server receives this message, it stops sending packets. When hand-offends, the node sends a message to the server that sets the window size to the value it had before handover. However, Freeze-TCP causes some adaptation problems. In upward handover, the node should wait to use full bandwidth. In downward hand-off, the node loses packets because of low bandwidth. One study has proposed improvements in Freeze-TCP are proposed [7] by calculating a new round trip time (RTT) for time-out solutions after hand-off. However, this method concentrates on the situation where hand-off has ended. Another proposed method is Freeze-TCP+ [8], which predicts bandwidth changes using the remaining packets for throughput after hand-off. However, hand-off start and end time are not defined. 
Multipath TCP (MPTCP) [21] is a set of extensions to regular TCP that allows one TCP connection to be spread across multiple paths. MPTCP distributes the load through the creation of separate sub-flows across potentially disjoint paths. A multipath flow should perform at least a single path flow on the best paths available to it, but it does not consider wireless networks, heterogeneous networks or hand-off [22].

Fast link adaptation (FLA) [11] is a method that can control the throughput using changed RTT information in vertical hand-off. However, FLA is designed to use changed RTT in hand-off. When vertical hand-off occurs, RTT changes rapidly, causing a congestion avoidance mode. Eventually, a node needs more time to adapt to the networks

The IEEE 802.21 MIH [6] standard defines media-access independent mechanisms that enable the optimization of hand-off between heterogeneous IEEE 802 systems and may facilitate hand-off between IEEE 802 systems and cellular systems. However, it still has some problems because it does not consider the transport layer hand-off [14].

\section{Freeze Dead Line Algorithm for Vertical Handoff in Heterogeneous Wireless Networks}

The use of Freeze-TCP resolves many hand-off problems of the transport layer in wireless networks; however, because Freeze-TCP is designed for horizontal networks, other problems occur when Freeze-TCP is used in heterogeneous wireless networks. These problems mainly involve the timing of the freezing and unfreezing of the networks.

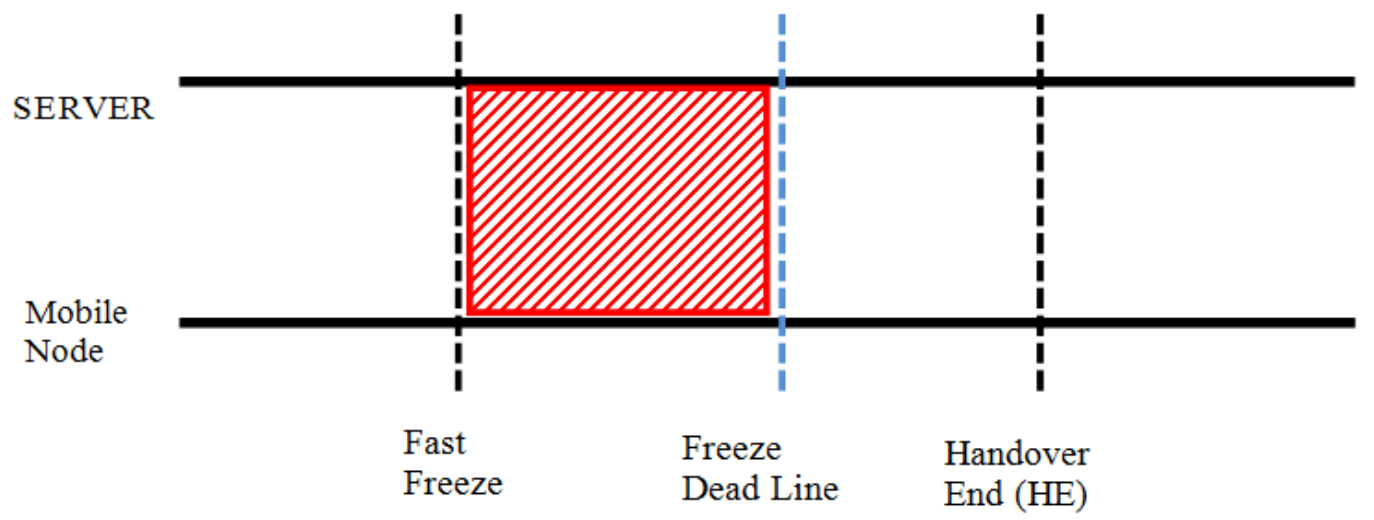

Figure 2. Fast Freeze

Figure 2 shows a fast freeze problem. If the freeze time is the FDL (Freeze Dead Line), then the fast freeze wastes packet transmission time. In Figure 2, deviant crease lines mean that if Freeze is normal, data transmission is possible; however, transmission is impossible because of the fast freeze. If the Freeze-TCP knows the exact freeze time, data are transmitted economically.

Figure 3 shows a late freeze problem. We assume that the packets transmitted the after FDL are all lost. If a mobile node sends a freeze request packet after the FDL, the packet lost because the server does not know if a hand-off occurred. The server therefore sends the packet to a mobile node, causing a timeout event. 


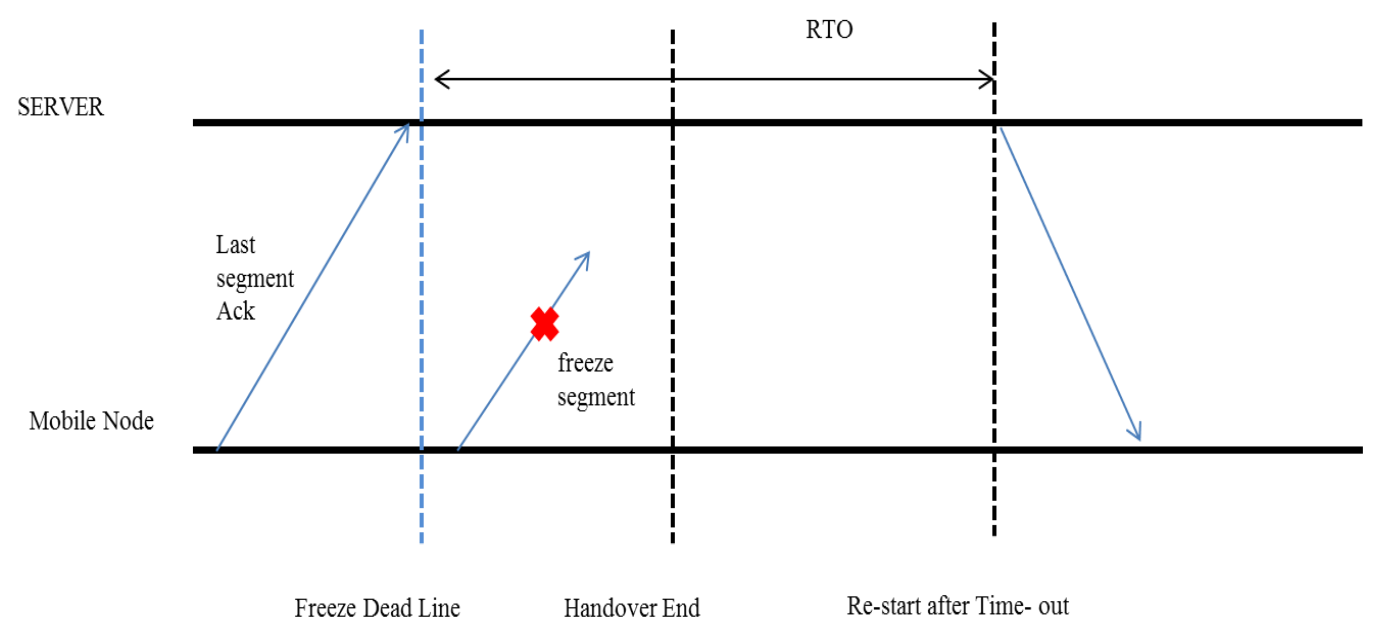

Figure 3. Late Freeze

We proposed the FDL method to solve this type of hand-off problem. We assume that the bottom layer sends messages that have a hand-off start time and hand-off end time. The MIH and cross layer methods are used to calculate the hand-off start and times. We also assume that all packets are lost from the hand-off start time to the hand-off end time

In this paper, FDL is a time at RTT/2 before the HE (hand-off end time) in current networks. It is described by the following equation:

$$
F D L_{\text {time }}=T_{\text {Handover Bnd }}-\frac{R T T_{\text {current }}}{2}
$$

Mobile nodes gather other network parameters and monitor the hand-off. If hand-off occurred, mobile node calculates the HE using past information, and sends a freeze request message to the server before the FLD. This is because the packet of the freeze request remains alive (We assume that all packets are lost from the hand-off start time to the hand-off end time) and safely arrives to server.

FDL shows better performance than other fast freeze methods. Suppose that the throughput is $\mathrm{G}$, where $\mathrm{G}$ is congestion window divided by RTT, as described by the following equation:

$$
G=\frac{C W N D}{R T T}
$$

The benefit of FDL is a given by the value of FDL time minus the early freeze time, multiplied by the throughput, $\mathrm{G}$, as is described by the following,

$$
F D L_{\text {benefit }}=\left(F D L_{\text {time }}-T_{\text {early Freeze }}\right) \times G
$$

Where $\mathrm{FLD}_{\text {time }}$ is hand-off start time and $\mathrm{T}_{\text {FastFreeze }}$ is the early Freeze time. The FDL $\mathrm{L}_{\text {benefit }}$ is always a positive number because the hand-off start time minus fast freeze time is greater than 0 (Equation 3), meaning that the FDL method has better performance than the fast freeze method. 


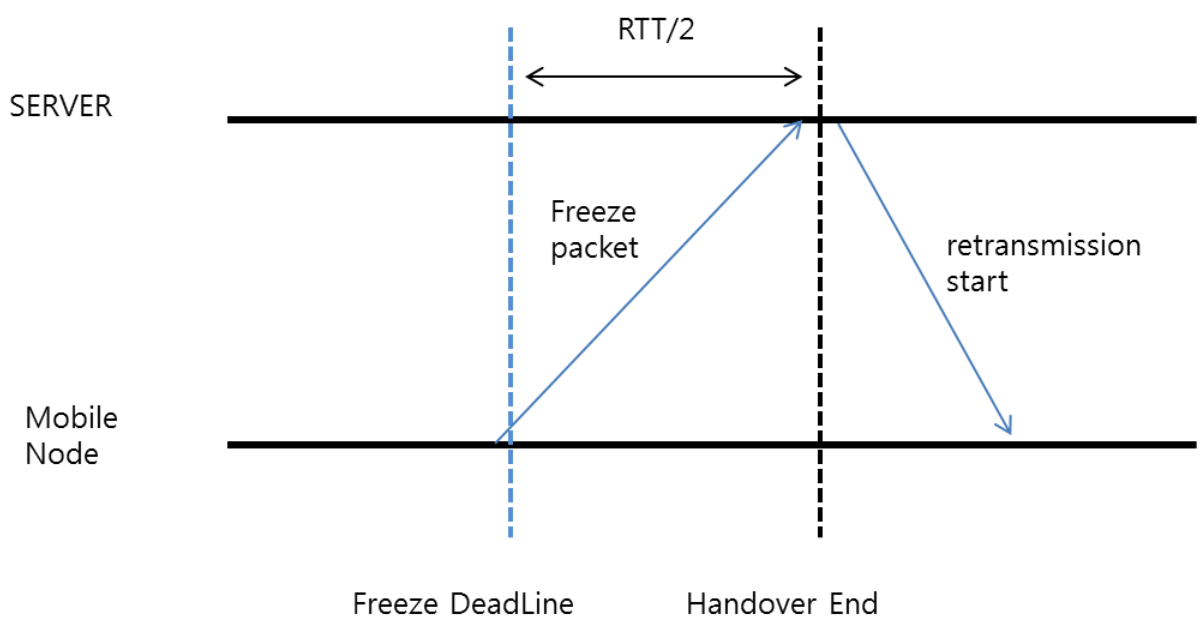

Figure 4. Propose Method

The proposed method is knows as hand-off end time using bottom layer information. When a mobile node sends a message of a network freeze, the mobile node sends a hand-off end time to the server in the FDL. However, the transport layer does not know whether a hand-off has occurred, in a late freeze situation, so, the server waits a long time from time of the last received ACK to when the time-out event occurs. FDL benefit is (equation 4) compare to the late freeze condition.

$$
F D L_{\text {benefit }}=\left(R T O-\left(T_{\text {handover end }}-F D L_{\text {time }}\right)\right) \times G
$$

Where RTO (Retransmission Timeout) is a time out term for the TCP, $\mathrm{T}_{\text {handoverEnd }}$ is the hand-off end time. The FDL benefit is shown Figure 5 compared to late freeze. In Figure 5, deviant crease lines indicate that the benefit of FDL compared to a late freeze. In late freeze, TCP does not know when the transmission started. Although the server can transmit the packets to the client, it does not do so; instead, it waits for the end of the time-out.

Another case is when a mobile node sends a hand-off end message after the hand-off end. However, this case also shows lower performance than FDL. A mobile node sends a message to the server after hand-off end, as shown in Figure 5. This message arrives via a new network after RTT/2(new network RTT) and the server resumes the transmission to the mobile node. However FDL resumes the transmission as soon as hand-off ends because the server knows the hand-off end time from the mobile nodes. This benefit is described by the following:

$$
F D L_{\text {benefit }}=\left(\frac{R T T_{\text {current }}}{2}\right) \times G
$$




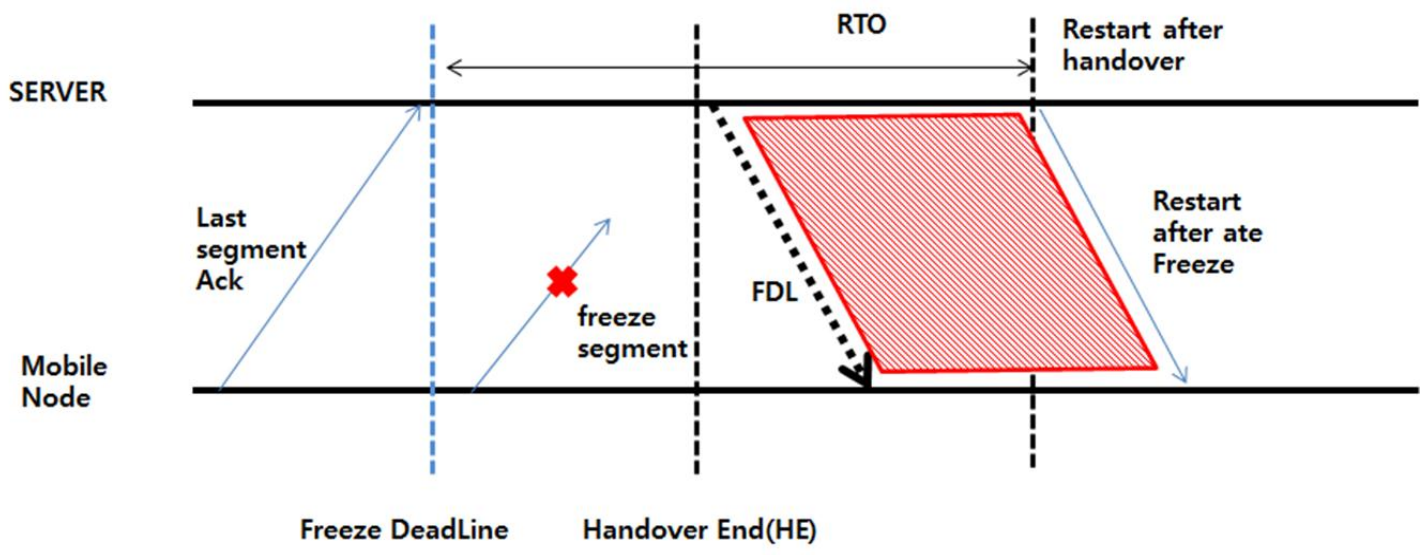

Figure 5. Late Freeze and FDL

RTT $_{\text {Current }}$ supposes a current RTT in new network. FDL sends a packet faster than the above case about RTT/2, confirming that the performance of the FDL is better than of that of other methods.

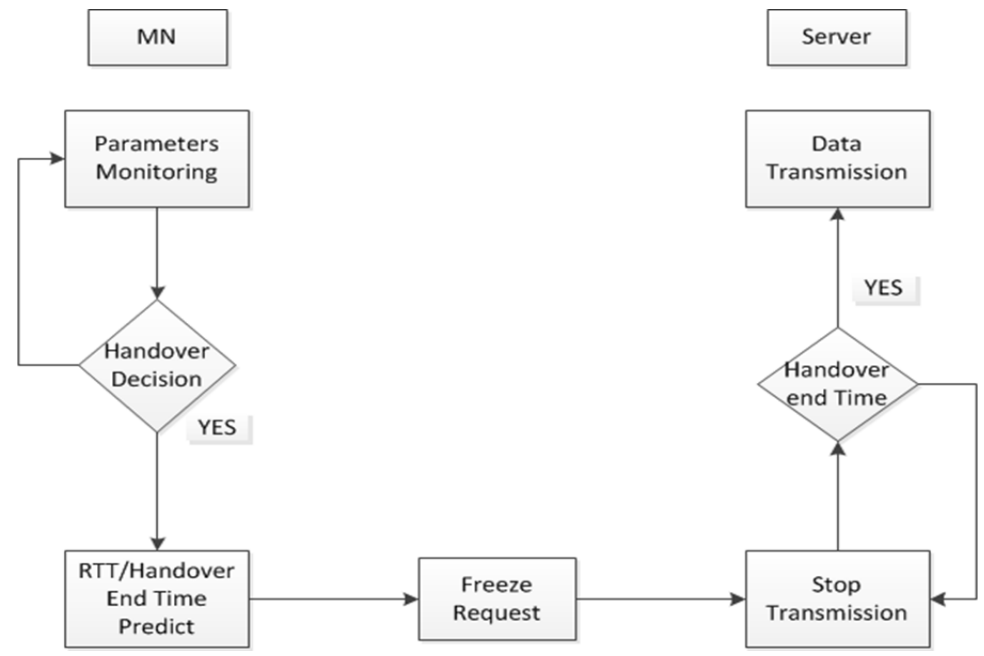

Figure 6. A Process of the FDL

Figure 6 shows a process of the FDL. A Mobile node monitors the parameters for hand-off. If a parameter is under the threshold, hand-off is initiated. The mobile node predicts the handoff end time using past information and sends it and the freeze request message to the server. The server receives the freeze request message, stops the transmission, and confirms the hand-off end time. If the hand-off end time has passed, it resumes the transmission. If the hand-off end time remains in the current time, the server wait until the hand-off end time and then resumes the transmission.

The mobile terminal has to know the hand-off end time (Thandoff end) in order to use FDL. Many methods are available; however, this paper uses past access information to predict the hand-off end time. We calculate the hand-off end time by using Information Service (MIIS) of the MIH. The MIIS has a function that sends the hand-off information from a mobile terminal to a networks hand-off. Much information is available for sending, [15] 


\begin{tabular}{|c|c|c|}
\hline General Information & Access Network Information & PoA Specific Information \\
\hline Operator ID & Network ID & \\
Network Type & Roaming Partners & PoA Link Addr \\
Service Provider ID & Cost & PoA Location \\
Country Code & Network QoS & PoA Channel range \\
$\ldots$ & Handoff Time & PoA System Info \\
& $\ldots$ & $\ldots$ \\
& $\ldots$ & \\
\hline
\end{tabular}

Figure 7. Parameters of MIIS

This paper introduces a new parameter, Handover Time(HT), which refers to the average time from hand-off start time to hand-off end time, which is described by the following equation:

$$
H T=\alpha \times H T_{\text {current }}+(1-\alpha) \times H T_{\text {old }}
$$

Figure 8 shows a flow chart of the FDL Process. The mobile node communicates the current network and the MIIS gathers information about the current and new networks (Information Update). To move from the current network to the new network, the mobile node requests new network information from the MIIS. The MIIS receives the mobile node's request and sends new network information. The MIIS also sends the HT. If the MIIS does not have new network information, it sends similar to information. The mobile node sends the information received from the MIIS such as HT or handover end time, to the server. The server receives the freeze request message, stops the transmission and confirms the hand-off end time. If the hand-off end time has passed, it resumes the transmission. If the hand-off end time is remains within the current time, the server waits until the hand-off end time and resumes the transmission.

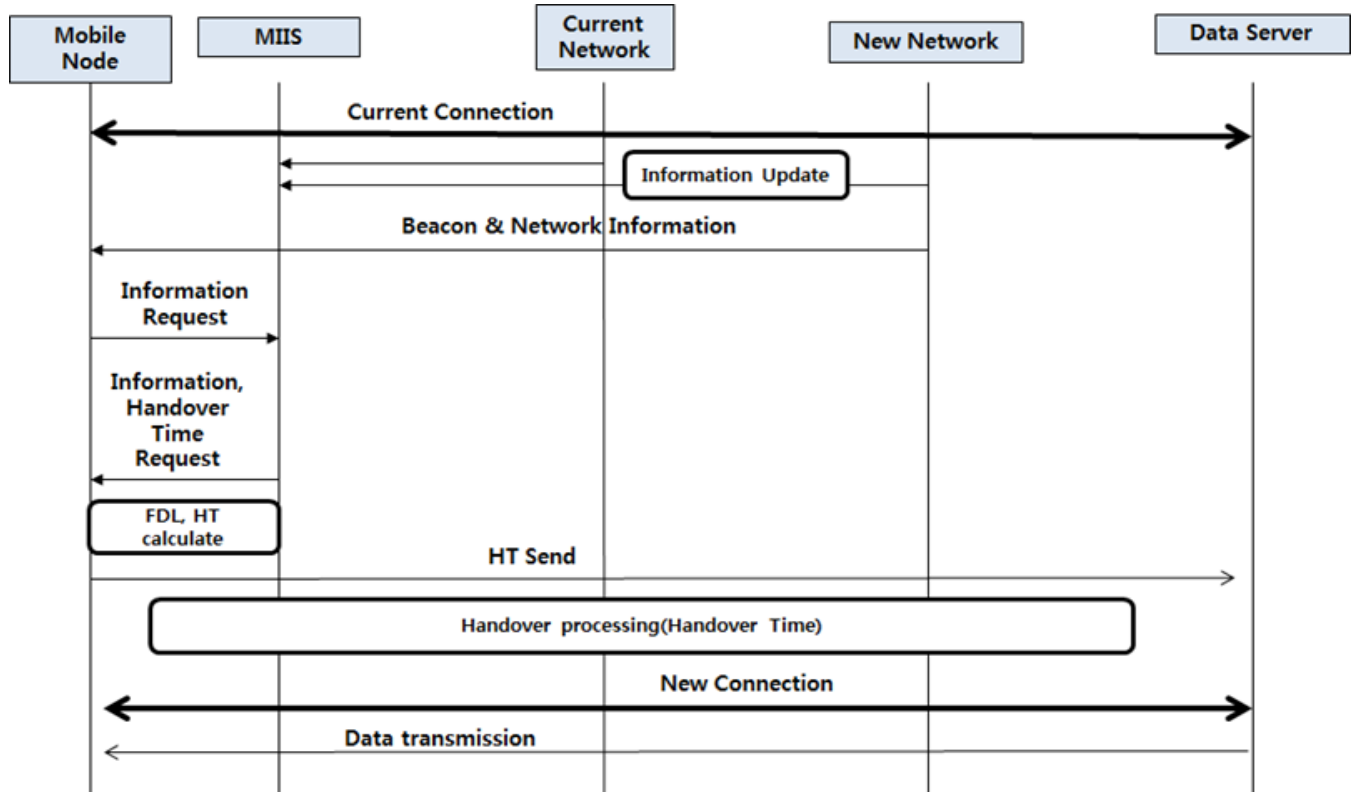

Figure 8. Flow Chart of the FDL Process 


\section{Experiment}

We simulated FDL in NS-2 (Network Simulator -2) [11]. Figure 8 shows and experimental environment consisting, of a server, a router, a base-station for a $3 \mathrm{G}$ network, and an access Point.

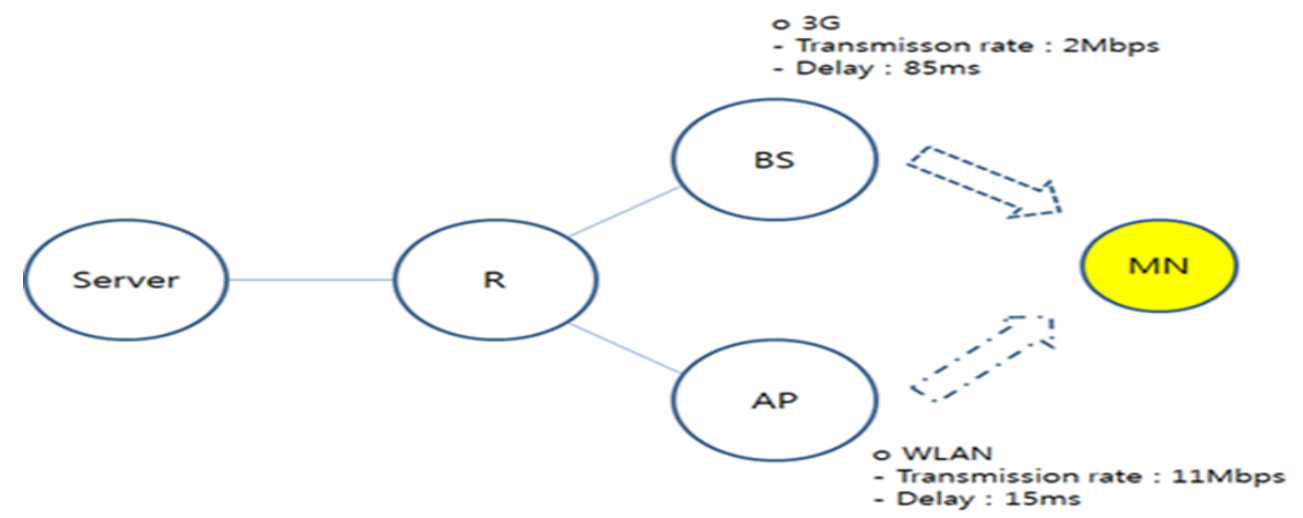

Figure 9. Simulation Topology

The server, router, basement (BS) and AP are connected with wires, while the mobile node and access point are connected wirelessly. After 10 seconds, a new network is established. The packet size is 1000 byte; it is used by FTP. The 3G bandwidth is $2 \mathrm{Mbps}$, with a delay of $85 \mathrm{~ms}$, and the WLAN bandwidth is $11 \mathrm{Mbps}$ with a $15 \mathrm{~ms}$ delay. We simulate four protocols, Freeze-TCP, TCP-Reno with applied FDL, TCP-Reno, and TCP-SACK. TCP-SACK is used in error environments and error rate is $1 \%$. We simulated two situations: a downward handoff(3G to WLAN) situation, and an upward hand-off (WLAN to 3G). We also measure the Congestion Control Window (CWND) to check the bandwidth adaptation after the hand-off. Parameters are drawn from related research $[12,13]$.

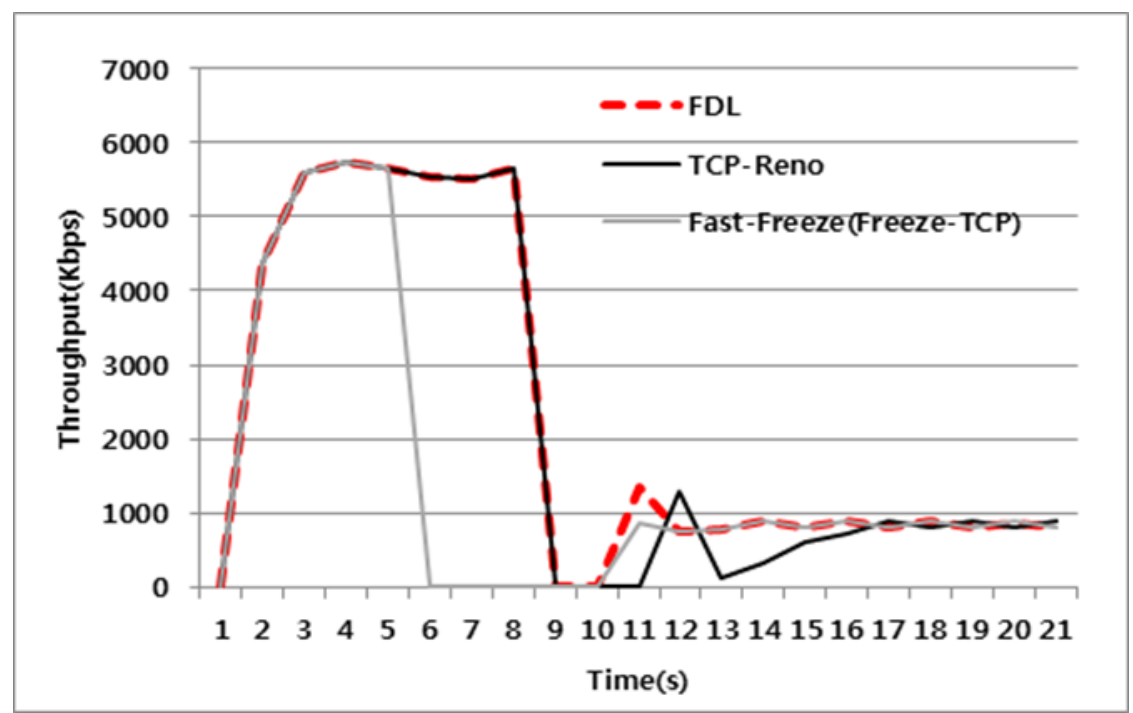

Figure 10. Throughput in Downward Vertical Hand-off

Figure 10 shows the throughput in the downward hand-off environment using FDL, TCPReno and Fast-freeze. After 5 seconds, we can see the fast freeze problem and that fast freeze 
causes bandwidth waste. Even though the server can transmit data, it does not do so because fast freeze has occurred. However, FDL knows the hand-off end time and when the last packet is sent, FDL continuously transmits packets. After 10 seconds from the simulation started, FDL transmits packets faster than TCP-Reno does. TCP-Reno does not know, when the hand-off started and ended. TCP know the last packet and RTO, so, TCP just perform a time out event. TCP transmits later than FDL.

Figure 11 shows throughput in the upward hand-off environment using FDL, TCP-Reno and Fast-freeze. After 5 seconds, we can see a fast freeze problem and fast freeze causes bandwidth waste. Even though the server can transmit data, it does not do so, because fast freeze has occurred. However, FDL work normally.

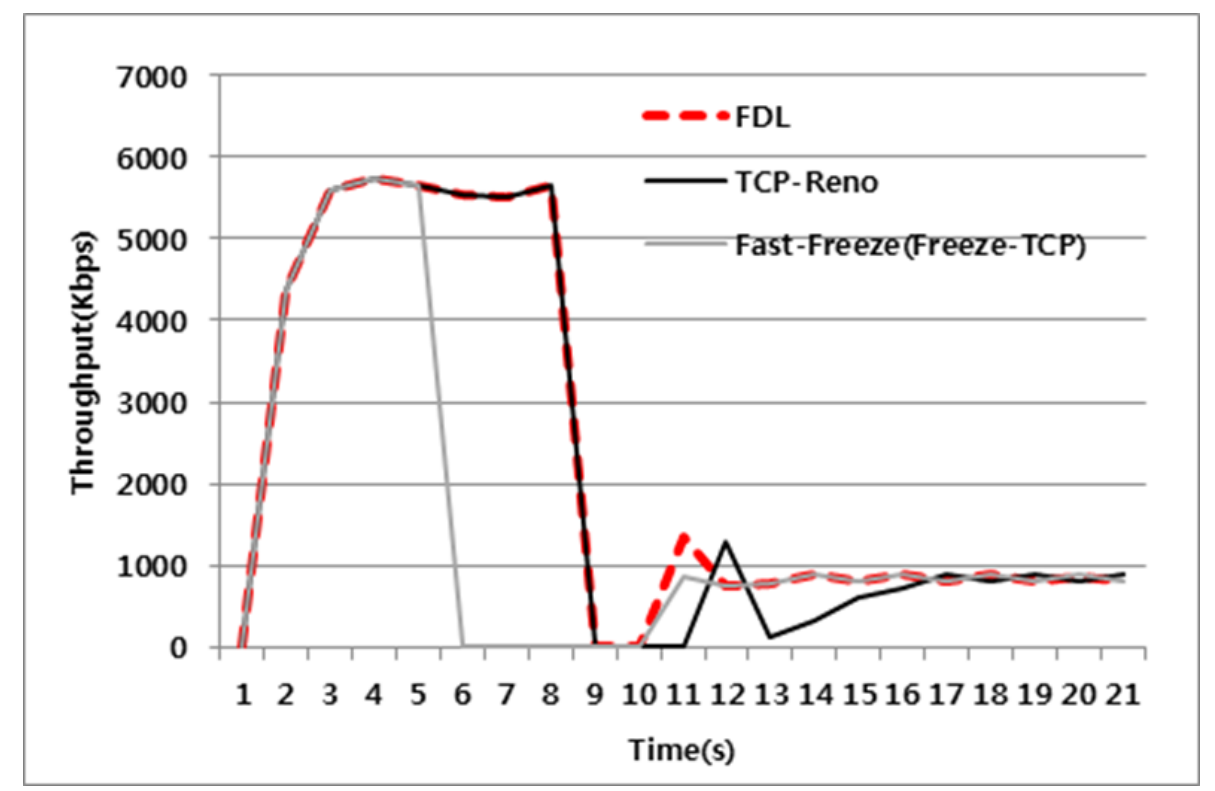

Figure 11. Throughput in Upward Vertcial Handoff

We simulated the error environment under same conditions as used in the above simulations. The purpose of the experiment is to investigate the real-world situation where many packet are lost due to diffraction, interference or collision. We set the error rate at about $1 \%$. Figure 12 shows an upward hand-off situation when packets are lost using TCP-SACK and FDL. The beginning of the graph Figure 11 shows a similar result with only different throughput; however, FDL has better more performance than TCP-Sack after the hand-off ended, because FDL knows the hand-off end time. 


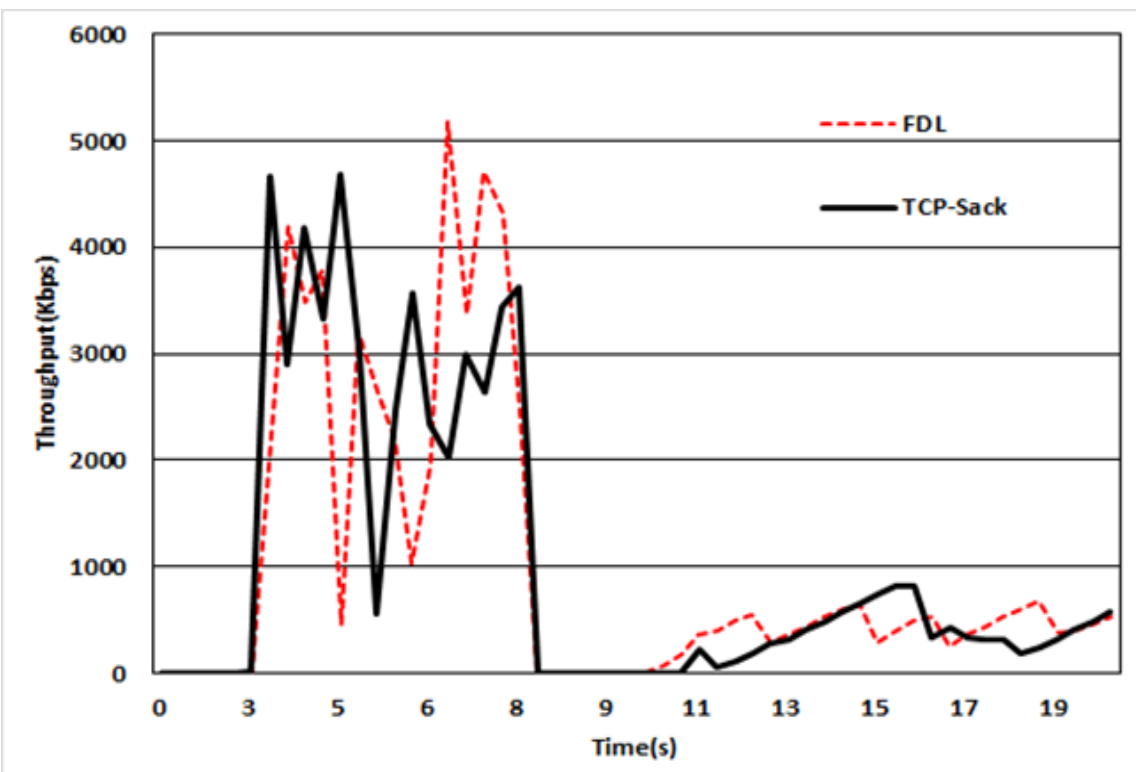

Figure 12. Throughput in Upward Handoff when Errors are occurred

Figure 13 shows downward hand-off situation when packet are lost using TCP-SACK and FDL. It also shows that FDL has better performance than TCP-SACK. FDL transmits a packet in advance of TCP-SACK, because FDL know the hand-off end time.

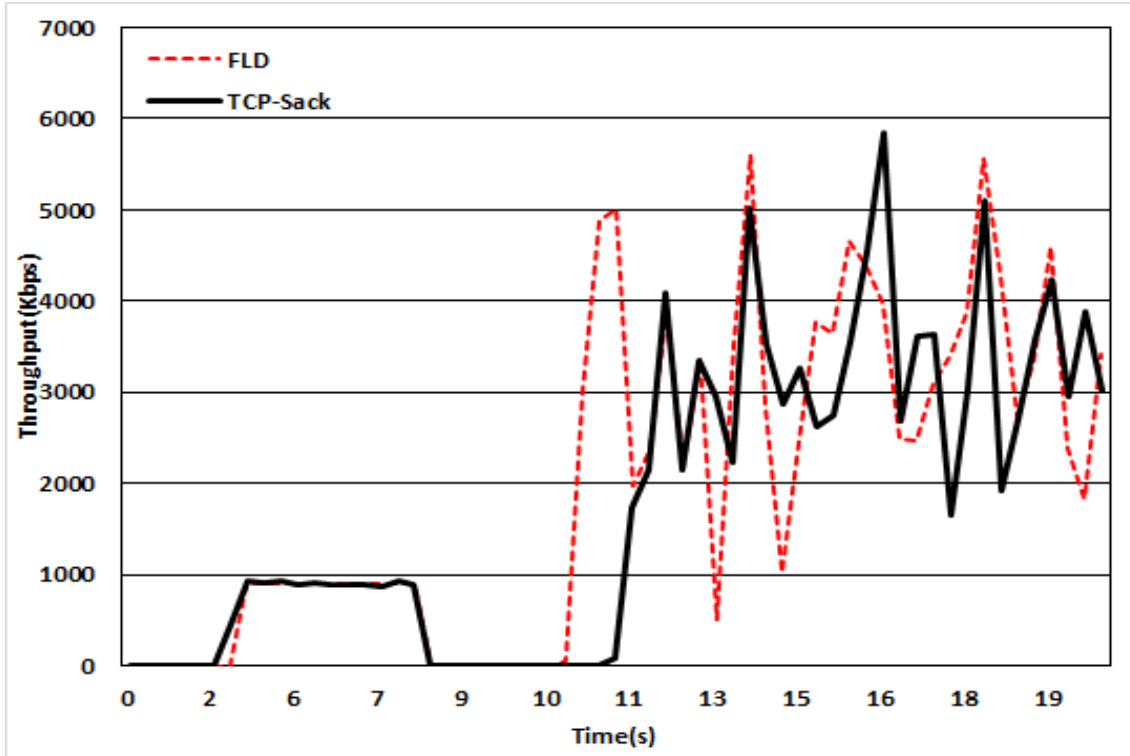

Figure 13. Throughput in Downward Hand off when Errors are occurred 


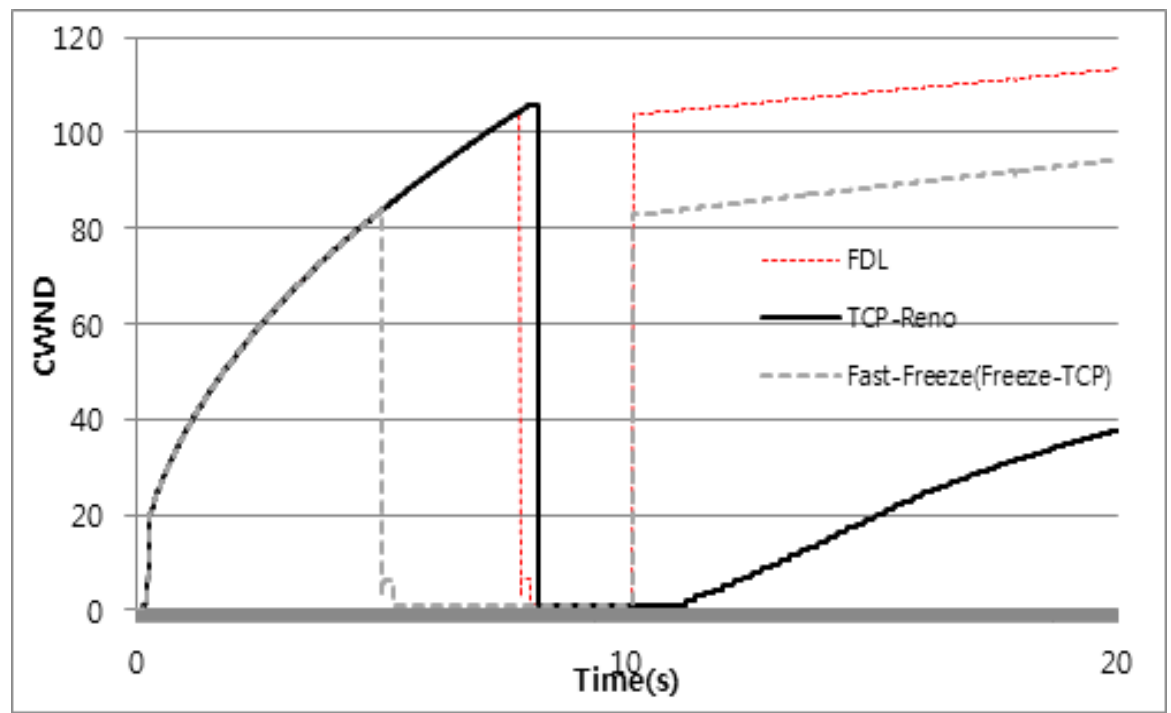

Figure 14. CWND in Upward Handoff

Figure 14 shows the congestion windows size in an upward hand-off situation. Fast freeze shows that CWND is 1 from 5 to 10 seconds. It causes late adaptation of the new network. Figure 15 shows that TCP-Reno CWND is 1 after the others hand-offs have ended. The proposed method maintains CWND at 0, but, FDL accomplishes a slow-start at the hand-off ended time. This enables the server to transmit a data earlier than TCP-Sack does.

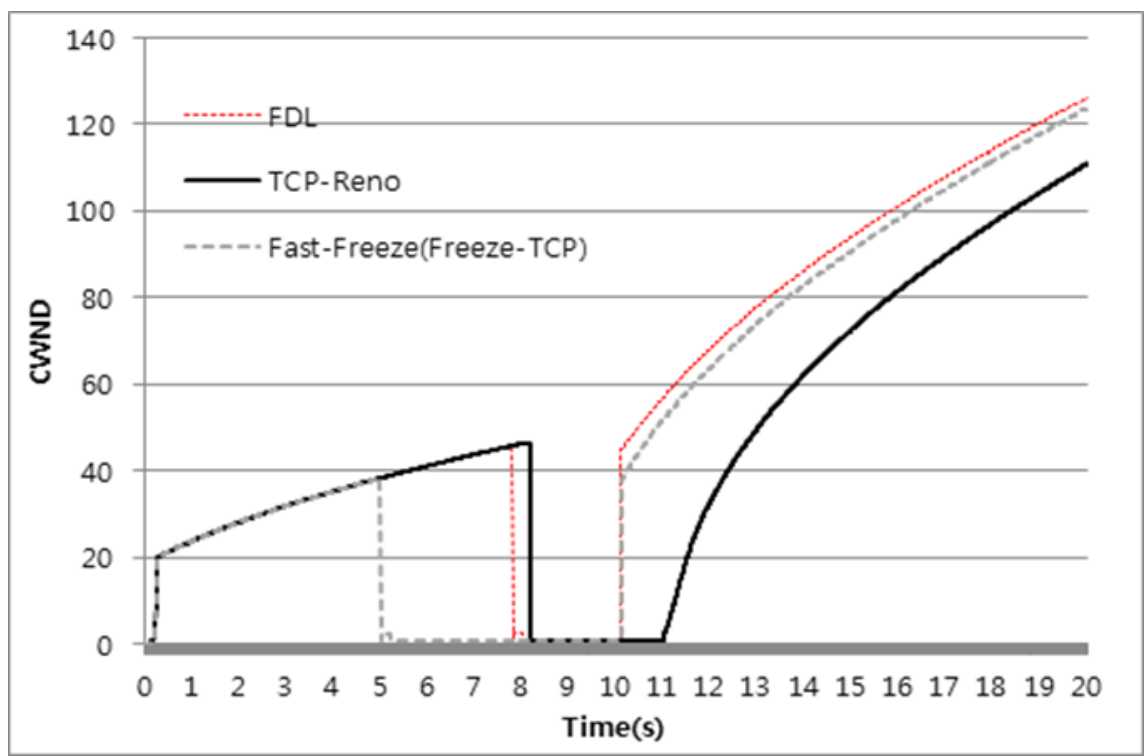

Figure 15. CWND in Downward Handoff

\section{Conclusion}

In heterogeneous wireless networks environments, hand-off problems have been considered from many aspects. However, recent researches has concentrated on the physical and link layer, whereas in this paper, we concentrated on transport layer. In a hand-off situation, the transport layer is sensitive to factors such as packet loss or throughput decreases, 
so solutions must be available to control these problems. The Freeze-TCP for the transport layer hand-off if one solution; however, freeze-TCP does not know when networks are freeze or unfreeze.

\section{Acknowledgements}

This work was supported by the Incheon National University Research Grant in 2013. This paper is a revised and expanded version of a paper entitled [Freeze Dead Line Method for Vertical Handover in Heterogeneous Wireless Networks] presented at [Advances in Net Generation Computer and Information Technology (NGCIT 2014), Vietnam, and October 2426 2014].

\section{References}

[1]. IEEE, "IEEE Standard for Local and metropolitan area network-Part 21: Media Independent Handover Services", IEEE std 802.21-2008, (2009), pp.1-90.

[2]. C. Lim and J. Jang, "A New Scheme to Improve Overall TCP Throughput with Vertical Handover between 3G Cellular Packet Networks and Wireless LANs", EURASIP Journal on Wireless Communications and Networking archive, vol. 2009, (2009).

[3]. H. Rutagemwa, S. Pack, X. Shen and J. Mark, "Robust Cross-Layer Design of Wireless-Profiled TCP Mobile Receiver for Vertical Handover", IEEE Trans Veh Technol., vol. 56, no. 6, (2007) November, pp. 3899-3911.

[4]. J. Fitzpatrick, S. Murphy, M. Atiquzzaman and J. Murphy, "Using cross-layer metrics to improve the performance of end-to-end handover mechanisms," Comput. Commun, vol. 32, no. 15, (2009), pp. 1600-1612.

[5]. H. Jeong and S Choi, "Transmission Rate Control in Heterogeneous Wireless Networks using Multiple Connections," Journal of the Korean institute of communication sciences, (2009), pp. 993-1003.

[6]. G. M. T. Da Costa and H. R. Sirisena, "Freeze TCP with time stamps for fast packet loss recovery after disconnections", Computer Communications, vol. 26, no. 15, (2003), pp. 1792-1799.

[7]. H. H. Lee and J. H Choi, "Enhanced Freeze-TCP for Vertical Hand-over", Conference on IEEK, vol. 2010, no. 6, (2010) May, pp. 887-889.

[8]. N. Wang, Y. Chen, C. Cheng, Y. Chiang and Y. Wang, "Improving TCP performance with fast adaptive congestion control during soft vertical handoff", Telecommun Syst, (2011).

[9]. E. Ko, D. An, I. Y. Yeom, "Congestion control for sudden bandwidth changes in TCP”, International Journal of Communication Systems, The Network Simulator NS-2, DOI:10.1002/dac.1322, (2011).

[10].S. Ko and K. Chung, "Rate Adaptation Scheme for Video Streaming Service in Vertical Handover Environments", Journal of KIISE: Information Networking, vol. 38, no. 6, (2011), pp. 439-449.

[11]. "IEEE Standard for Local and metropolitan area networks - Part 21: Media Independent Handover Services," (2009) January.

[12].G. Tim, M. James, D. S. Phatak, G. Vipul, "Freeze - TCP: A true end-to-end TCP enhancement mechanism for mobile environments", in Proc. IEEE INFOCOM, (2000) March, pp. 1537-1545, Israel.

[13].D. Li, K. Sleurs, E. Lil and A. Capelle, "Fast link adaptation for TFRC after a handover", Proc. of the IEEE International Symposium on Personal, Indoor and Mobile Radio Communications, (2008), pp. 1-5.

[14].V. Tiwari, S. Kansal and A. Gaiwak, "Performance evaluation of TCP variants using media independent handover in heterogeneous network," Proc .of the International Conference on Computer and Communication Technology, (2010) September, pp. 367-370.

\section{Authors}

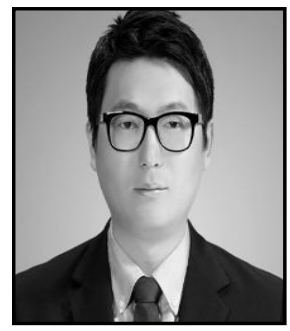

Hyeon-Jin Jeong, He received the M.S degree in computer science from Incheon National University, South Korea, in 2012. His research interests include human-computer interaction for wireless network protocol, routing protocol. 


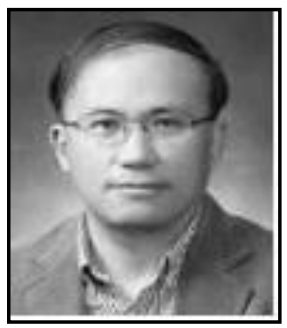

Seung-Sik Choi, He received the M.S. and Ph.D. degree from KAIST, Korea, in 1990 and 2002, respectively. He had worked for Korea Telecom Research Center from 1990 to 2002. He is a professor in Department of Computer Science and Engineering at Incheon National University. His current research topics are access control and internet protocol in wireless networks 
International Journal of Control and Automation Vol. 7, No. 12 (2014) 